\title{
Experimental Study and Modeling of Silicon Supersaturated with Selenium by Ion Implantation and Nanosecond-Laser Melting
}

\author{
F.F. Komarov ${ }^{a, *}$, G. Ivlev ${ }^{b}$, G. Zayats ${ }^{c}$, A. Komarov $^{a}, \mathrm{~N} \mathrm{NeChaeV}^{a}$, \\ I. PARKhomenko ${ }^{b}$, L. Vlasukova ${ }^{b}$, E. WendleR $^{d}$ And S. MiskieWicz $^{a}$ \\ ${ }^{a}$ A.N. Sevchenko Institute of Applied Physical Problems, Belarusian State University, \\ Kurchatov Str. 7, 220045 Minsk, Belarus \\ ${ }^{b}$ Belarusian State University, Nezavisimosti Ave. 4, 220030 Minsk, Belarus \\ ${ }^{c}$ Institute of Mathematics, National Academy of Sciences of Belarus, \\ Surganov Str. 11, 220072 Minsk, Belarus \\ ${ }^{d}$ Friedrich-Schiller University Jena, Max-Wien-Platz 1, 07743 Jena, Germany
}

\begin{abstract}
Selenium supersaturated silicon is a promising material for intermediate-band solar cells and extended infrared photodiodes. Selenium-rich Si layers were fabricated by Se ion implantation followed by pulsed laser melting using one or three pulses. The Rutherford backscattering spectrometry in random and channeling directions, the Raman spectroscopy, and photoluminescence techniques were used to study structural and optical properties of the Se-rich silicon layers. It is shown that laser irradiation leads to silicon recrystallization and significant impurity redistribution in the implanted layer. According to the Rutherford backscattering data, the substitutional fraction of Se atoms after laser treatment is $60-80 \%$. The analysis of photoluminescence spectra revealed that pulsed laser irradiation of the implanted layer with the power density of $1.5 \mathrm{~J} / \mathrm{cm}^{2}$ leads to the formation of vacancy and interstitial Si clusters. After annealing at the power density higher than $1.5 \mathrm{~J} / \mathrm{cm}^{2}$, the photoluminescence originating from vacancies and interstitials disappears. To explain the evolution of the Se distribution within the implanted layer after laser melting, numerical solution of the 1D diffusion equations was used.
\end{abstract}

DOI: 10.12693/APhysPolA.136.254

PACS/topics: silicon, Se ion implantation, pulse laser annealing, RBS, PL

\section{Introduction}

Silicon is the basic semiconductor material for technologies of a great number of micro- and optoelectronic devices, including conventional Si photodetectors for visible and near-infrared radiation (IR) with $\lambda<1.1 \mu \mathrm{m}$. Extending the spectral sensitivity of such detectors to the short-wavelength IR spectral region $(\lambda=1.4-3.0 \mu \mathrm{m})[1]$ is of great importance for optical communication, integrated optoelectronics, solar cells technology and other applications. Chalcogenhyperdoped silicon with the reduced optical band gap down to $0.5 \mathrm{eV}$ is a very attractive material for nearinfrared applications.

Si doped with chalcogens ( $\mathrm{S}, \mathrm{Se}, \mathrm{Te}$ ) of the concentration much above the equilibrium solid solubility exhibits strong sub-bandgap absorption. As a result of the sub-bandgap formation in chalcogen doped $\mathrm{Si}$, the broad short-wavelength infrared absorption edge is shifted down to $0.5 \mathrm{eV}[1,2]$, which is an important characteristic for development of IR photodetectors.

*corresponding author; e-mail: komarovf@bsu.by
Se hyperdoped Si shows also insulator-to-metal transition (the Mott transition) and metallic-like conduction behaviour [3, 4].

Pulsed laser irradiation is known as an effective method for chalcogen-hyperdoping of silicon. For instance hyperdoping of $\mathrm{Si}$ by Se-impurity can be obtained using the pulsed laser mixing $[5,6]$ or by incorporation of S-dopant into Si during the fs-laser ablation of $\mathrm{Si}$ in the $\mathrm{SF}_{6}$ atmosphere [7, 8]. A very attractive method for the formation of hyperdoped Si with dopant concentration exceeding the equilibrium solid solubility by the orders of magnitude rely on ion implantation followed by the nanosecond pulsed laser melting (PLM) [9]. Although this method has been known for several decades, it attracted a renewed interest for the formation of chalcogen-supersaturated Si layers $[2,3,9,10]$.

In this paper the research data obtained on the basis of a new combination of experimental methods and PLM numerical modeling are presented. In order to study the influence of PLM parameters on the dynamics of the laser action, depth distribution and lattice location of the Se impurity atoms, structural and optical properties of the laser-modified layers a nanosecond ruby laser was used. The optical diagnostics of PLM was controlled by monitoring the time-resolved reflectivity (TRR), while 
the implanted Si was investigated by the Raman spectroscopy, photoluminescence, the Rutherford backscattering spectrometry in random $(\mathrm{RBS} / \mathrm{R})$ and channeling $(\mathrm{RBS} / \mathrm{C})$ directions.

\section{Experimental}

Silicon (111) $p$-type wafer with the resistivity $\rho=10 \Omega \mathrm{cm}$ was implanted with $125 \mathrm{keV}{ }^{80} \mathrm{Se}^{+}$to a fluence of $1 \times 10^{16}$ ions $/ \mathrm{cm}^{2}$. Pulsed laser irradiation of the implanted layers was carried out under the experimental conditions described in Refs. [11, 12], i.e. with the diagnostics (by means of TRR measurements at $\lambda=1.06 \mu \mathrm{m})$ of solid $\leftrightarrow$ liquid phase transitions induced in the sample by the pulsed laser irradiation $(\lambda=0.69 \mu \mathrm{m}, 70 \mathrm{~ns}$ FWHM). The pulse energy densities $W$ were a set at $1.5,2.0$, and $2.5 \mathrm{~J} / \mathrm{cm}^{2}$. For each $W$, two implanted samples were irradiated with one or three laser shots. Se depth distribution profiles, substitutional fraction of incorporated Se in a Si lattice, and Si crystallinity of the implanted and annealed samples were analyzed by $\mathrm{RBS} / \mathrm{R}$ and $\mathrm{RBS} / \mathrm{C}$. These measurements were performed using $1.4 \mathrm{MeV} \mathrm{He}{ }^{+}$ions with the normal incidence and $170^{\circ}$ scattering angle. The Raman spectroscopy (RS) and photoluminescence (PL) spectra were obtained to analyze structural peculiarities of the Se supersaturated Si layers. The RS measurements were performed in backscattering geometry with a Nanofinder High End micro-Raman spectrometer (LOTIS TII) using a $473 \mathrm{~nm}$ laser beam as the excitation source at room temperature. PL was investigated at low temperature $(4.2 \mathrm{~K})$ using the $\mathrm{He}-\mathrm{Ne}$ laser beam $(633 \mathrm{~nm})$ with an excitation power density $70 \mathrm{~mW} / \mathrm{cm}^{2}$. The dispersed luminescence was detected by the cooled InGaAs detector. The modeling of impurity redistribution caused by laser melting and subsequent solidification was performed using the program code made by the authors.

\section{Results and discussion}

Under the present experimental conditions, the threshold value for the laser-induced $\mathrm{Si}$ surface melting is $\approx 0.3 \mathrm{~J} / \mathrm{cm}^{2}$. Table I presents the value of the melt lifetime $\tau_{m}(W)$, which is the duration of the laserinduced solid $\leftrightarrow$ liquid phase transitions and the maximum thickness $d_{m}(W)$ of the liquid Si layer (depth of fusion) depending on the ruby laser radiation energy density $W$. The $\tau_{m}(\mathrm{~W})$ were measured by the TRR method. The corresponding depth of fusion values was evaluated using the model described in Ref. [13].

The $\mathrm{RBS} / \mathrm{R}$ and $\mathrm{RBS} / \mathrm{C}$ spectra for the asimplanted $\mathrm{Si}$ and laser-treated with single pulse of $W=2.5 \mathrm{~J} / \mathrm{cm}^{2}$ samples are presented in Fig. 1 . The depth distribution profiles of Se in Si calculated from the RBS-spectra using an algorithm presented in Ref. [14] for the as-implanted and laser-treated samples are shown in Fig. $2 \mathrm{a}$ and $\mathrm{b}$.
TABLE I

Si melt lifetime $\tau_{m}(W)$ and thickness $d_{m}(W)$ of melted layer formed by ruby laser pulse with energy density $W$.

\begin{tabular}{c|c|c|c}
\hline \hline $\mathrm{W}\left[\mathrm{J} / \mathrm{cm}^{2}\right]$ & 1.5 & 2.0 & 2.5 \\
\hline$\tau_{m}[\mu \mathrm{s}]$ & 0.17 & 0.27 & 0.35 \\
$d_{m}[\mu \mathrm{m}]$ & 0.2 & 0.4 & 0.6
\end{tabular}

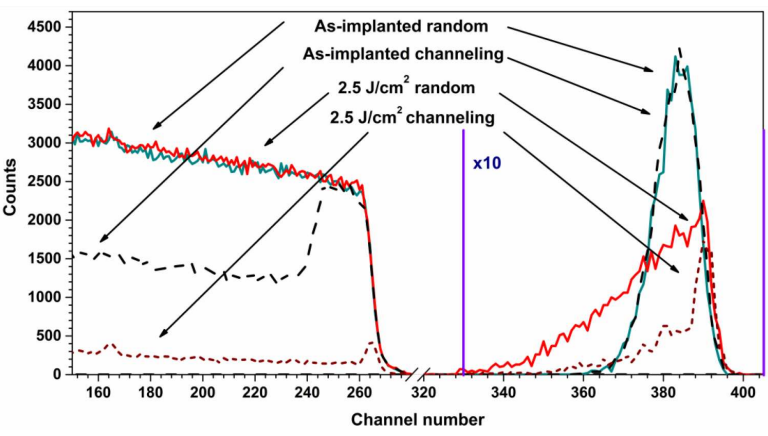

Fig. 1. RBS/R and RBS/C spectra of the asimplanted and annealed by a ruby laser pulse $\left(W=2.5 \mathrm{~J} / \mathrm{cm}^{2}, \tau=70 \mathrm{~ns}\right)$.
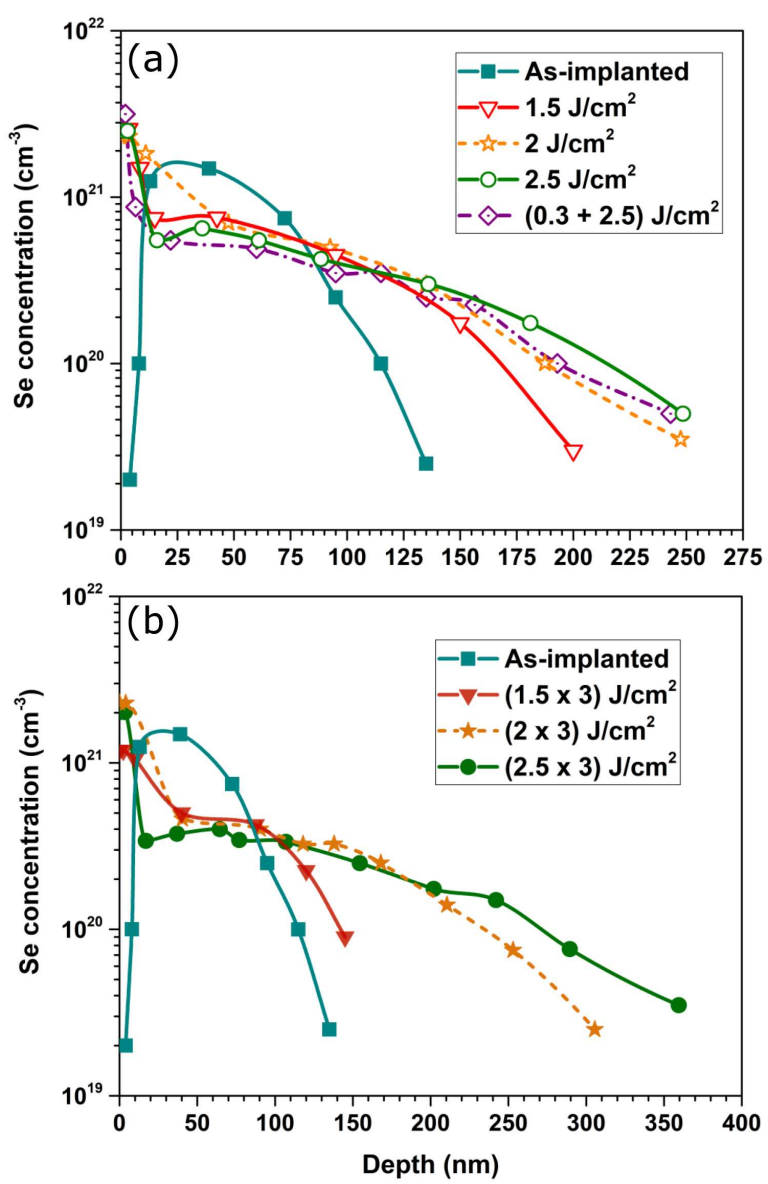

Fig. 2. Depth profiles calculated from RBS/R spectra of Se concentration in Si matrix after ion implantation $\left(125 \mathrm{keV}, 1 \times 10^{16} \mathrm{~cm}^{-2}\right)$ and subsequent pulsed laser irradiation $(0.69 \mu \mathrm{m}, 70 \mathrm{~ns})$ with an energy density varied from 1.5 to $2.5 \mathrm{~J} / \mathrm{cm}^{2}$ with 1 shot (a) and 3 shots (b). 
Pulsed laser melting leads to a significant redistribution of the impurity up to a depth of 300-400 nm with the formation of a decreasing plateau with the Se concentration nearly $\left(3 \times 10^{19}-5 \times 10^{20}\right) \mathrm{cm}^{-3}$ and accumulation of impurity in the near-surface layer of around $20 \mathrm{~nm}$ thick. The Se concentration in the layer at the depth of 50-120 nm does not change significantly for all samples presented in Fig. 2. For the laser pulse energy density of 2.0 and $2.5 \mathrm{~J} / \mathrm{cm}^{2}$ and three pulses, Se diffuses more deeply into the sample. After one laser pulse of $1.5 \mathrm{~J} / \mathrm{cm}^{2}$, the thickness of the layer containing Se atoms is $\approx 200 \mathrm{~nm}$ which is the same as that of the molten layer (Table I). By increasing the number of pulses with $W=1.5 \mathrm{~J} / \mathrm{cm}^{2}$ to three, the thickness of the layer containing Se atoms is reduced to $\approx 150 \mathrm{~nm}$. It seems that recrystallization front during the solidification pushes the impurity towards the surface. For all presented samples, the thickness of the layer containing Se atoms with the concentration higher than RBS detection limit $\left(\approx 5 \times 10^{18} \mathrm{~cm}^{-3}\right)$ does not exceed the calculated thickness of the molten layer formed by laser irradiation.

The information about silicon matrix crystallinity and concentration of Se atoms incorporated into the silicon matrix was obtained via comparison of the aligned and random RBS spectra. Here, the minimum yields $\chi_{\min }^{\mathrm{Si}}$ and $\chi_{\mathrm{min}}^{\mathrm{Se}}$ are defined as the ratio of the backscattering yield of channeling to that of random spectra for silicon and selenium atoms, respectively. $\chi_{\min }^{\mathrm{Si}}$ exhibits the overall level of the lattice quality [15]. Fully amorphous sample has $\chi_{\min }^{\mathrm{Si}}$ equal to $100 \%$ and a high-quality crystal has $\chi_{\min }^{\mathrm{Si}}$ as low as $1-5 \%$. Figure 3 presents estimations of the substitutional rate profiles of Se atoms as $100 \%-\chi_{\min }^{\mathrm{Se}}(\mathrm{z})$. The inset in Fig. 3 shows the total substitutional fraction $f$ of Se atoms after different annealing regimes calculated using Eq. (1) from Ref. [15]. Thus,

$$
f=\left(100 \%-\chi_{\min }^{\mathrm{Se}}\right) /\left(100 \%-\chi_{\min }^{\mathrm{Si}}\right) .
$$

The as-implanted silicon is fully amorphous in the Se atoms containing region $\left(\chi_{\min }^{\mathrm{Se}}=100 \%, \quad \chi_{\min }^{\mathrm{Si}}=100 \%\right.$ at the depth of $0-150 \mathrm{~nm})$. Deeper than $150 \mathrm{~nm}$, $\chi_{\min }^{\mathrm{Si}}=56 \%$ (Fig. 1). This indicates that either Si matrix is largely damaged or the analyzing $\mathrm{He}$ ions are dechanneled while passing the amorphous surface layer. Pulsed laser annealing with the power density larger than the threshold for the melting leads to silicon recrystallization. It was detected that $\chi_{\min }^{\mathrm{Si}}=(11 \pm 5) \%$ in all samples after the laser treatment. Most of Se atoms $(60-80) \%$ are incorporated into the silicon lattice. The silicon amorphization after the ion implantation and the damaged layer recrystallization after PLM are also confirmed by RS (not shown). The sample irradiated with the laser power density of $0.31 \mathrm{~J} / \mathrm{cm}^{2}$ prior to the second pulse of $2.5 \mathrm{~J} / \mathrm{cm}^{2}$ reveals a slightly different RBS spectrum from the sample irradiated with just a single shot of $2.5 \mathrm{~J} / \mathrm{cm}^{2}$. This "pre-heated" sample is characterized with the maximum substitutional fraction of Se $(87 \%)$ and the most efficient silicon matrix recrystallization $\left(\chi_{\min }^{\mathrm{Si}}=5 \%\right)$.

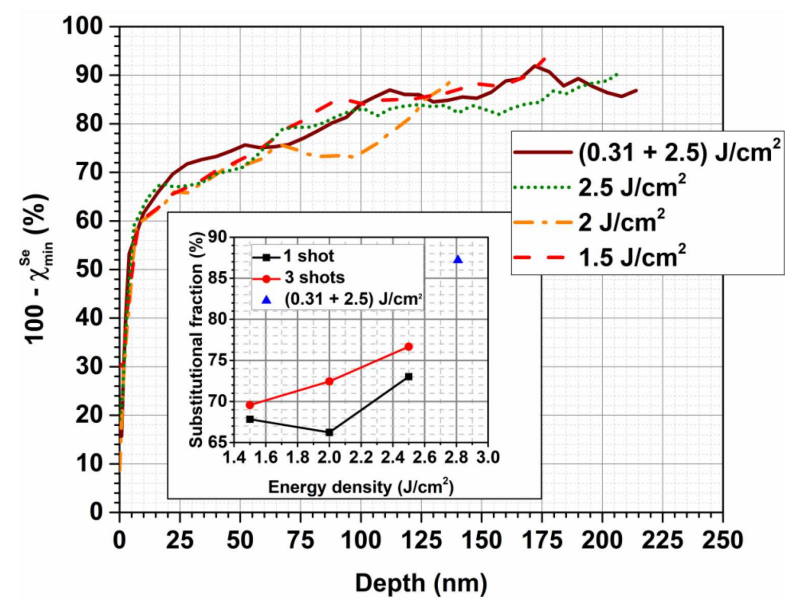

Fig. 3. Depth profiles of the substitutional rate of Se as $100 \%-\chi_{\min }^{\mathrm{Se}}(z)$. The inset shows the total substitutional fraction of incorporated Se in Si lattice under different annealing conditions calculated from Eq. (1).

Figure 4 shows the low temperature photoluminescence spectra of the as-implanted and laser treated samples. In the as-implanted PL spectrum, two weak bands appear at 1415 and $1528 \mathrm{~nm}$. This coincides with the D1 and D2 lines related to the dislocation luminescence. Laser irradiation with the energy density $W=1.5 \mathrm{~J} / \mathrm{cm}^{2}$ results in the appearance of a weak band at $\approx 1190 \mathrm{~nm}(\mathrm{X}$ line $)$ and an intense narrow band at $\approx 1216 \mathrm{~nm}$ (W line) with the series of their phonon replicas in the luminescence spectrum. The $\mathrm{W}$ and $\mathrm{X}$ centers consist of Si self-interstitial clusters [16-18]. The W center has trigonal symmetry while the $\mathrm{X}$ center has tetragonal symmetry [16-18]. An increase in the number of pulses to three at an energy density of $1.5 \mathrm{~J} / \mathrm{cm}^{2}$ leads to a decrease in the intensity of the $\mathrm{X}$ and $\mathrm{W}$ lines and to an increase in the intensity of the bands D1 and D2 (Fig. 4b). An increase in the pulse energy up to $2 \mathrm{~J} / \mathrm{cm}^{2}$ leads to a decrease in the intensity of dislocation lines whereas the $\mathrm{W}$ line is very weakly manifested. With an increase in the number of $2 \mathrm{~J} / \mathrm{cm}^{2}$ pulses to three, the $\mathrm{W}$ line disappears completely, and on the contrary, the intensity of the dislocation luminescence bands increases. In the spectrum of the sample irradiated with a single pulse of the energy density $2.5 \mathrm{~J} / \mathrm{cm}^{2}$, the D1 and D2 lines have nearly the same intensity as in the as-implanted sample. With increasing the number of $2.5 \mathrm{~J} / \mathrm{cm}^{2}$ pulses to three, only the dislocation luminescence band D1 is weakly manifested. Thus, it can be concluded that the as-implanted sample contains dislocations (apparently under the amorphous implanted layer). The significant decrease in the $\mathrm{W}$ line intensity as well as the increase of the band edge $\left(\mathrm{FE}_{\mathrm{TO}}\right)$ line with the increasing pulse energy and the number of laser pulses suggest the recreation of crystal quality of implanted layer. It should be noted that the sample irradiated with 0.3 and $2.5 \mathrm{~J} / \mathrm{cm}^{2}$ pulses results in the best crystal quality within the Se-rich layer. 

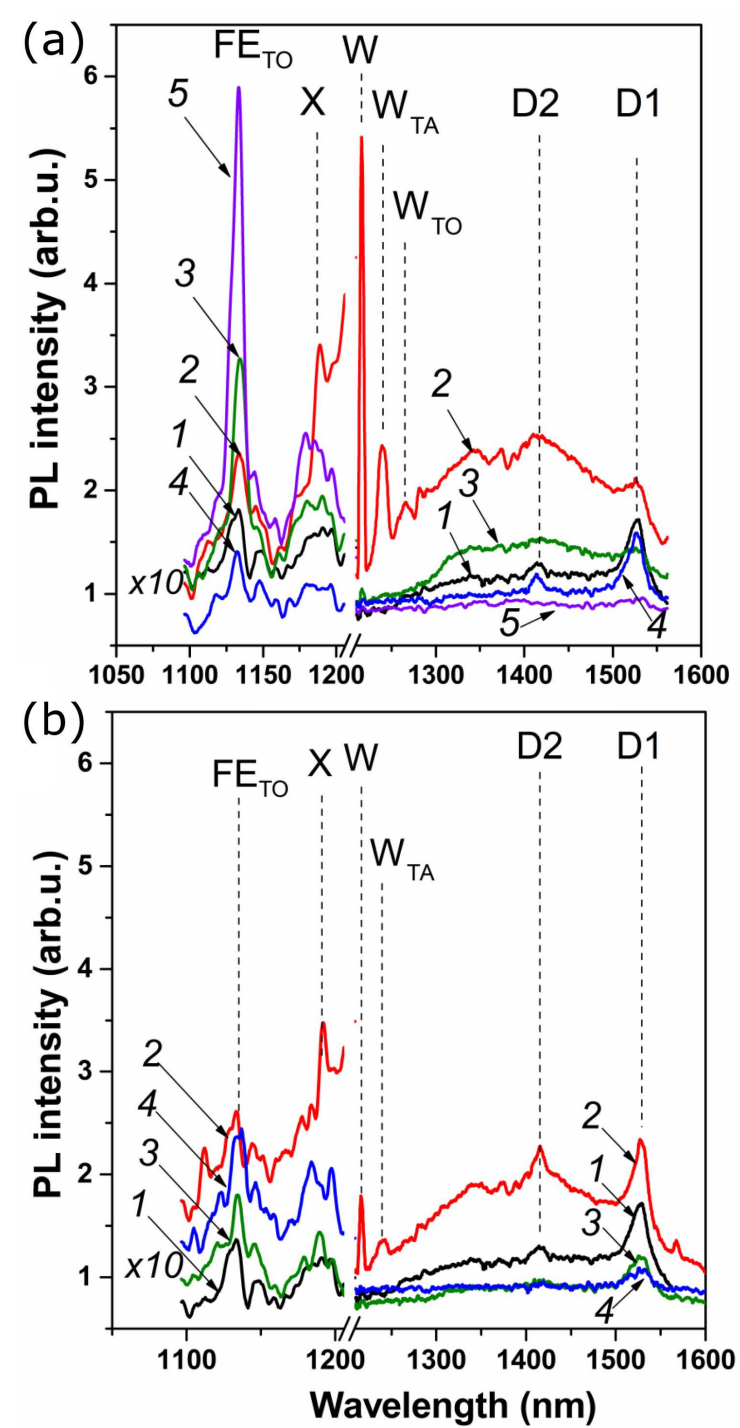

Fig. 4. PL spectra of Se-implanted silicon before (1) and after pulsed laser melting with (a) one and (b) three pulses at energy densities $W$ of $1.5(2), \quad 2(3), \quad 2.5 \mathrm{~J} / \mathrm{cm}^{2}(4)$ and combination of $(0.3+2.5) \mathrm{J} / \mathrm{cm}^{2}(5)$.

To understand the evolution of the Se concentration profile during laser melting and solidification, a numerical solution of the 1D diffusion equations was used. Segregation of the impurity was taken into account as the factor which leads to violation of the continuity of particles separation at the solid-liquid interface and the impurity displacement by the crystallization front to the surface.

The mathematical description of the impurity diffusion process is based on the use of the Fick second law. The equations of impurity diffusion in the liquid (L) and solid (S) phases of silicon are presented in the following form:

$$
\frac{\partial C_{L}}{\partial t}=D_{L} \frac{\partial^{2} C_{L}}{\partial x^{2}}, \quad 0<x<x_{\text {front }}(t),
$$

$$
\frac{\partial C_{S}}{\partial t}=D_{S} \frac{\partial^{2} C_{S}}{\partial x^{2}}, \quad x_{\text {front }}(t)<x<l,
$$

with the boundary conditions

$$
\left.D_{L} \frac{\partial C_{L}}{\partial x}\right|_{x=0}=0,\left.\quad D_{S}\right|_{x=l}=0 .
$$

The value of the right boundary must be chosen to be sufficiently large so that the second condition in Eq. (3) is valid. The initial conditions is

$$
C_{0}=C_{\text {impl }}(x), \quad 0 \leq x \leq l .
$$

On the movable solid-liquid front $x=x_{\text {front }}(t)$, the defined condition for the continuity of the impurity flux and the concentration matching condition are, respectively

$$
\begin{aligned}
& \left.D_{L} \frac{\partial C_{L}}{\partial x}\right|_{x=x_{\text {front }}(t)}=\left.D_{S} \frac{\partial C_{S}}{\partial x}\right|_{x=x_{\text {front }}(t)}, \\
& \left.C_{S}\right|_{x=x_{\text {front }}(t)}=\left.k_{\text {segr }} C_{L}\right|_{x=x_{\text {front }}(t)} .
\end{aligned}
$$

In the system of Eqs. (2)-(5), $C_{L}, C_{S}$ are the impurity concentrations, $D_{L}, D_{S}$ are the diffusion coefficients of the impurity for the liquid and solid phases of the silicon substrate, respectively, $t$ is the time, $x$ is the spatial coordinate, $C_{\mathrm{impl}}(x)$ is the initial impurity concentration profile, $k_{\text {segr }}$ is the impurity segregation coefficient, and $x=x_{\text {front }}(t)$ is the crystallization front (the time dependence of the coordinate of the interface between the solid and liquid phases of silicon).

In the presented model, impurity diffusion is simulated in the liquid and solid phases. In each stage of the crystallization process, the ratio of concentrations in the solid and liquid phases at the interface is determined by the value $k_{\text {segr }}$. The impurity that is not embedded in the lattice accumulates at the solid-liquid interface and diffuses into the remaining melt region in accordance with the law defined by the diffusion Eq. (2a), and deep into the crystal according to the law determined by the diffusion Eq. (2b).

The system of Eqs. (2)-(5) is numerically solved by the finite-difference method, according to which the differential equations are replaced by the discrete analogues obtained on the difference grid [19]. At each time step the solution of the constructed system of linear algebraic equations with a three-diagonal matrix is found by the Thomas algorithm [19].

The results presented in Ref. [13] are used to describe the movable solid-liquid interface $x=x_{\text {front }}(t)$. The velocity of the solid-liquid front is easy to estimate, when the thickness of the molten area and its lifetime $\tau_{p}^{\max }$ are known. A typical value of speed of the melting front in $\mathrm{Si}$ induced by a nanosecond range laser pulse is $10 \mathrm{~m} / \mathrm{s}$. During subsequent solidification, the solid-liquid front typically moves towards the surface with the velocity of 1-2 m/s. Both values are given for the substrate temperature of $300 \mathrm{~K}[20]$. In our case $v_{k r}=1.9 \mathrm{~m} / \mathrm{s}$. The diffusion coefficient of Se in the liquid phase is given by the constant value $D_{L}=2.4 \times 10^{-4} \mathrm{~cm}^{2} / \mathrm{s}$. $D_{L}$ is calculated using the algorithm described in Ref. [21]. That is the same order of magnitude as the diffusion coefficients for the other elements in the molten Si [22]. 

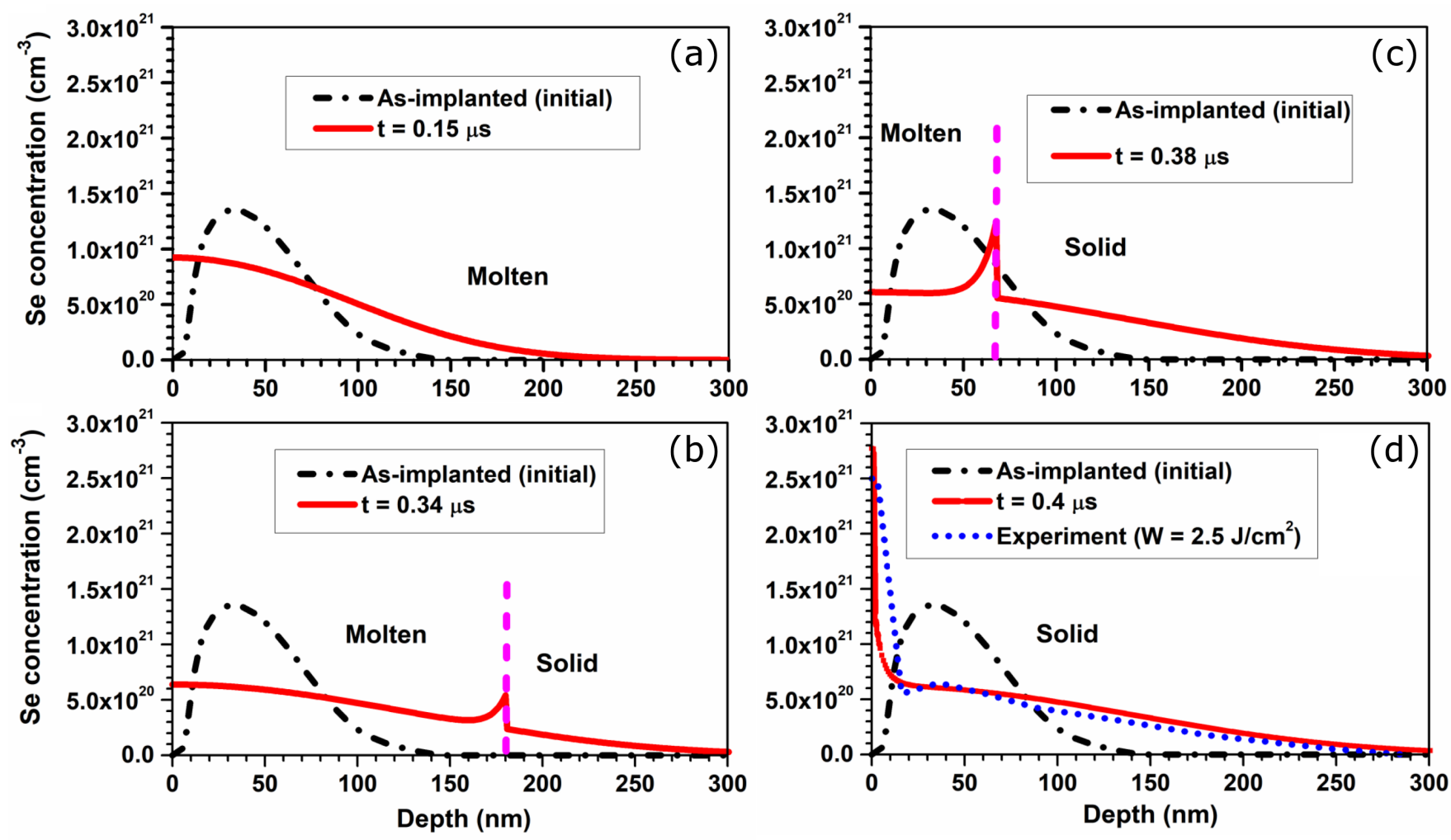

Fig. 5. Redistribution dynamics $(t=0.15 \mu \mathrm{s}(\mathrm{a}), 0.34 \mu \mathrm{s}(\mathrm{b}), 0.38 \mu \mathrm{s}(\mathrm{c})$, and $0.4 \mu \mathrm{s}(\mathrm{d}))$ of implanted Se in Si induced by ruby laser pulse $\left(W=2.5 \mathrm{~J} / \mathrm{cm}^{2}, \tau=70 \mathrm{~ns}\right)$. Dashed-dotted line is as-implanted concentration profile, solid lines-calculated profiles and dotted line-experimental profile.

The diffusion coefficient in the solid phase $D_{S}$ in this model is given by the mean (fitted to the experiment) value, $D_{S}=5 \times 10^{-5} \mathrm{~cm}^{2} / \mathrm{s}$. In the present model the temperature dependence has not been embedded yet, although it is assumed that the temperature in the solid and the liquid phases are close to the crystallization temperature of Si molten layer which was measured as $1410^{\circ} \mathrm{C}$ [11]. The value of the segregation coefficient was determined by modelling with the requirement of the best matching of the solution to the experimental profile, $K_{\text {segr }}=0.15$. The experimental Se profile in asimplanted was taken as $C_{\text {impl }}(x)$. The modelling continues until the interface reaches $10 \mathrm{~nm}$ from the surface. It is assumed that the impurity remaining in this layer accumulates on the surface and that is when the calculation stops.

Figure 5a and b present the simulation of the selenium redistribution during annealing by the ruby laser pulse with the energy density of $W=2.5 \mathrm{~J} / \mathrm{cm}^{2}$. The dynamics of impurity redistribution is shown as a function of the motion of the solid-liquid front. When the laser induced melting is over, broadening of the Se distribution profile is observed (Fig. 5a). Further, in the initial stage of solidification (Fig. 5b), Se, which is not incorporated into the $\mathrm{Si}$ lattice, is pushed towards the surface by the moving solid-liquid front. The segregation process develops as the interfacial boundary moves towards the surface (Fig. 5c). At the final stage of the process (Fig. 5d), a concentration peak of selenium is formed (in the near-surface layer with a thickness of about $20 \mathrm{~nm}$ ). The resulting calculated selenium depth distribution is in good agreement with the experimental data.

\section{Conclusions}

It has been shown that pulsed laser irradiation of selenium implanted silicon leads to a significant impurity redistribution and most of Se atoms (up to 87\%) are incorporated into silicon matrix. The mathematical model for selenium redistribution in the silicon matrix during nanosecond pulse laser melting was developed. Good agreement between the modelled and experimental profiles of selenium depth distribution in silicon after laser melting allowed to determine the diffusion coefficient of selenium in the solid phase during solidification (crystallization front moves to the surface). In future, it is supposed to introduce a functional dependence of the diffusion coefficients of the impurity, both in the liquid phase and in the solid on temperature of the annealed region of the semiconductor, which is determined from the heat equation [13].

The sample pre-heated by $0.31 \mathrm{~J} / \mathrm{cm}^{2}$ laser pulse, and subject to subsequent irradiation by $2.5 \mathrm{~J} / \mathrm{cm}^{2}$ pulse is 
characterized with the maximum substitutional fraction of Se $(87 \%)$ and the most efficient silicon matrix recrystallization $\left(\chi_{\min }^{\mathrm{Si}} \approx 5 \%\right)$.

In low-temperature PL spectrum of as-implanted sample, two dislocation-related weak bands appear at 1415 and $1528 \mathrm{~nm}$ (D1 and D2 lines). Such dislocations are apparently formed below the amorphous implanted layer. A subsequent laser treatment is followed with the appearance of $\mathrm{X}(\approx 1190 \mathrm{~nm})$ and $\mathrm{W}(\approx 1216 \mathrm{~nm})$ lines which are assigned to self-interstitial clusters. Moreover, intensity of dislocation-related PL bands as well as $\mathrm{X}$ and $\mathrm{W}$ lines are very sensitive to the laser irradiation. After three laser pulses with the power density of $2.5 \mathrm{~J} / \mathrm{cm}^{2}$, only the dislocation luminescence band D1 is weakly manifested.

\section{References}

[1] Y. Berencén, S. Prucnal, F. Liu, et al., Sci. Rep. 7, 43688 (2017).

[2] I. Umezu, J.M. Warrender, S. Charnvanichborikarn, A. Kohno, J.S. Williams, M. Tabbal, D.G. Papazoglou, X. Zhang, M.J. Aziz, J. Appl. Phys. 113, 213501 (2013).

[3] E. Ertekin, M.T. Winkler, D. Recht, A.J. Said, M.J. Aziz, T. Buonassisi, J.C. Grossman, Phys. Rev. Lett. 108, 026401 (2012).

[4] S. Hi, P. Han, P. Liang, Y. Xing, S. Lou, Mater. Sci. Semicond. Process. 17, 134 (2004).

[5] M. Tabbal, T. Kim, D.N. Woolf, B. Shin, M.J. Aziz, Appl. Phys. A 98, 589 (2010).

[6] B.K. Newman, E. Ertekin, J.T. Sullivan, M.T. Winkler, M.A. Marcus, S.C. Fakra, M. Sher, E. Mazur, J.C. Grossman, T. Buonassisi, J. Appl. Phys. 114 133507 (2013).

[7] M.A. Sheehy, L. Winston, J.E. Carey, C.M. Friend, E. Mazur, F.Y. Genin, Chem. Mater. 17, 3582 (2005).
[8] V. Zobra, N. Boukos, I. Zergioti, C. Fotakis, Appl. Opt. 47, 1846 (2008).

[9] W. Yang, J. Mathews, J.S. Williams, Mater. Sci. Semicond. Process. 62, 103 (2017).

[10] B. Bob, A. Kohno, S. Charnvanichborikarm, J.M. Warrender, I. Umezu, M. Tabbal, J.S. Williams, M.J. Aziz, J. Appl. Phys. 107, 123506 (2010).

[11] G.D. Ivlev, E.I. Gatskevich, Appl. Surf. Sci. 143, 265 (1999).

[12] H.A. Novikov, R.I. Batalov, R.M. Bayazitov, I.A. Faizrakhmanov, G.D. Ivlev, S.L. Prokop'ev, Tech. Phys. 60, 405 (2015).

[13] V.A. Pilipovich, V.L. Malevich, G.D. Ivlev, V.V. Zhidkov, J. Eng. Phys. 48, 228 (1985).

[14] N.P. Barradas, C. Jeynes, R.P. Webb, Appl. Phys. Lett. 71, 291 (1997).

[15] L.C. Feldman, J.W. Mayer, S.T. Picraux, Materials Analysis by Ion Channeling: Submicron Crystallography, Academic Press, New York 1982.

[16] M. Abboy, I. Santos, P. López, L.A. Marques, L. Pelaz, J. Electron. Mater. 47, 5045 (2018).

[17] V.D. Tkachev, C. Schrödel, A.V. Mudryi, Radiat. Eff. 49, 133 (1980).

[18] P. Giri, Semicond. Sci. Technol. 20, 638 (2005).

[19] A. Samarskii, E. Taft, Z. Nashed, The Theory of Difference Schemes, CRC Press, Boca Raton 2001.

[20] W.L. Brown, Laser and Electron Beam Processing of Materials, Academic Press, New York 1980.

[21] H.S. Carslaw, J.C. Jaeger, Conduction of Heat in Solids, Clarendon Press, Oxford 1959.

[22] D.K. Belashchenko, Transport Phenomena in Liquid Metals and Semiconductors, Atomizdat, Moscow 1970 (in Russian). 